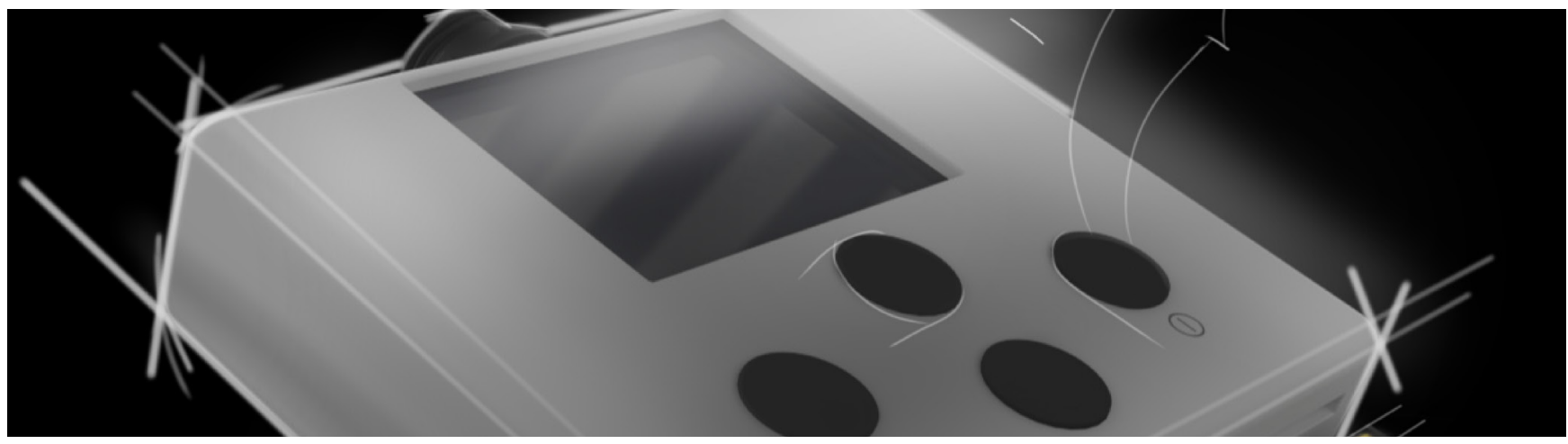

\title{
Desarrollo de un bioimpedancímetro portable para pacientes con enfermedad renal crónica y deportistas de alto rendimiento
}

Development of a portable bioimpedance monitor system for Chronic Kidney Disease Patients and high performance athletes

Andrés Felipe Pineda-Alpízar ${ }^{1}$, Federico González-Camacho² ${ }^{2}$ Javier Reina-Tosina ${ }^{3}$, David Naranjo-Hernández ${ }^{4}$, Gerardo Barbarov-Rostán ${ }^{5}$

A.F. Pineda-Alpízar, F. González-Camacho, J. Reina-Tosina, D. Naranjo-Hernández, G. Barbarov-Rostán “Diseño industrial y desarrollo de un bioimpedancímetro portable”, IDI+, vol. 3, no 2, Ene., pp. 31-45, 2021.

doi https://www.doi.org/ 10.18845/ridip.v3i2.5564

Fecha de recepción: 20 de febrero de 2020 Fecha de aprobación: 10 de noviembre de 2020

1. Andrés Felipe Pineda-Alpízar Estudiante de Ingeniería en Diseño Industrial. Instituto Tecnológico de Costa Rica Cartago, Costa Rica fpineda147@outlook.com (D) 0000-0003-2095-6926

2. Federico González-Camacho Escuela de Diseño Industrial. Instituto Tecnológico de Costa Rica Cartago, Costa Rica fgonzalez@tec.ac.cr (D) 0000-0002-0180-1585
3. Javier Reina-Tosina Grupo de Ingeniería Biomédica, Escuela Técnica de Ingeniería, Universidad de Sevilla

Sevilla, España jreina@us.es

(D) 0000-0002-6853-4899

4. David Naranjo-Hernández Grupo de Ingeniería Biomédica, Escuela Técnica de Ingeniería, Universidad de Sevilla Sevilla, España dnaranjo@us.es

(D) 0000-0002-7636-2855

5. Gerardo Barbarov-Rostán Grupo de Ingeniería Biomédica, Escuela Técnica de Ingeniería, Universidad de Sevilla Sevilla, España gbarbarov@gmail.com

(D) 0000-0003-4383-9394 


\section{Resumen}

El presente artículo da cuenta de la investigación para la innovación, diseño y desarrollo industrial de un dispositivo médico portable que obtiene datos relevantes de la bioimpedancia humana con precisión clínica, desarrollado en el Grupo de Ingeniería Biomédica de la Universidad de Sevilla en conjunto con el Tecnológico de Costa Rica para su diseño industrial. Los sistemas de bioimpedancia son una saliente novedosa en el campo de la e-salud, sin embargo, el incremento de la expectativa de vida y la inclusión de tecnología de monitoreo en aplicaciones deportivas han aumentado la necesidad de dispositivos médicos más portables, precisos y comerciables a nivel masivo, con el objetivo de empoderar al usuario sobre su situación médica. El método utilizado fue una investigación cualitativa centrada en el usuario mediante una metodología guía desarrollada por el autor, el modelo Divergente-Convergente Proyectual (DGC) centrado en el usuario. Los hallazgos más importantes fueron el diseño novedoso de un bioimpedancímetro portable para pacientes con afecciones renales crónicas y deportistas de alto rendimiento, así como el uso de una metodología novedosa para el desarrollo de productos médicos portables.

\section{Palabras clave}

Diseño; dispositivo médico; E-Health; enfermedad renal crónica; dispositivo de monitoreo.

\section{Abstract}

The following article presents the research and innovation in the industrial design and development of a portable bioimpedance monitor system with clinical precision developed by the Biomedical Engineering Group of the University of Seville and with the Costa Rica Institute of Technology regarding the industrial design. Bioimpedance systems possess industrial design challenges for its application in the e-health field, even though, the continuous increase in life expectancy and the need for more precise medical equipment for sport applications have risen the need for portable and precise medical equipment that can be mass scaled commercialized and empower the user's healthcare situation. A qualitative approach method was used with a methodology designed by the author (Divergent-Convergent Project (DGC) Method). The most important findings were the industrial design and development of the bioimpedance monitor system and the novel methodology for the development of novel portable medical products.

\section{Keywords}

Design; medical device; E-Health; chronic kidney disease; monitoring device. 


\section{Introducción}

\section{Problemática General}

El sistema de salud actual está condicionado a una alta dependencia de sistemas de monitoreo no portables que influyen directamente en la capacidad de respuesta hospitalaria [1]. Durante los últimos años, un incremento en la e-salud y sus diversas aplicaciones han ido en crecimiento y aplicación en distintos campos, específicamente con el objetivo de hacer sostenibles los sistemas públicos de salud ante el crecimiento de enfermedades crónicas, envejecimiento de la población y la demanda de mejores estándares de calidad en la población general [2].

La enfermedad renal crónica (ERC) es definida como la reducción de la tasa de filtración glomerular, incremento de la excreción de la albúmina urinaria o ambas y se considera un problema de salud pública de alto riesgo hoy en día, además, se estima que la prevalencia de la ERC a nivel mundial es de un $8 \%$ a un 16\% [4]. En el caso de los pacientes con enfermedad renal crónica, la valoración de una composición corporal puede ser crítica para la adecuación de las terapias renales sustitutivas [3]. Entre las complicaciones que generan, se pueden enumerar los problemas cardiovasculares, enfermedad del riñón progresiva, falla aguda del riñón, declive cognitivo, entre otros [4]. El monitoreo, revisión constante y utilización de sistemas de alarma pueden facilitar el tratamiento y monitoreo de progresión de la enfermedad renal crónica y en los lugares donde se han realizado planes de contingencia se ha logrado reducir el desarrollo de la etapa final de la enfermedad [5].

El desarrollo de la bioimpedancia en el mercado deportivo profesional es otra saliente de la tecnología que está tomando mucha importancia, tal como lo es la aplicación específica al fútbol, específicamente a los estudios desarrollados a jugadores profesionales de la Serie A de la Liga profesional italiana [6]. Otro aspecto importante es la división de la bioimpedancia para casos de estudio específicos, tales como [7]:

- Uso de datos específicos del análisis de bioimpedancia [7].

- Comparación de los cambios en el análisis de bioimpedancia entre atletas de distinto género y disciplina deportiva [6].

- Cambios de la composición corporal durante breves y extensos lapsos de ejercicio bajo intensidades cambiantes [6].

- Diferencias de la composición corporal entre atletas de una misma disciplina, pero labora distinta (Deportes por equipo) [6].

Es así como diversas aplicaciones de la bioimpedancia se desarrollan en mercados específicos y emergentes, sin embargo, se requieren para la correcta aplicación de la técnica, condiciones estandarizadas de medición [1]. 


\section{Bioimpedancia como herramienta diagnóstica}

La técnica de la bioimpedancia consiste en la inyección de una corriente alterna eléctrica de muy baja intensidad en un medio biológico (cuerpo humano, tejido biológico o cultura celular), esto con el objetivo de detectar caídas de voltaje en el medio; a mayor la caída respectiva, mayor la impedancia eléctrica presentada en el medio [21]. Las medidas de bioimpedancia se basan en el hecho de que los medios biológicos se comportan como conductores, dieléctricos o aislantes a la corriente eléctrica respectiva [21].

El desarrollo de la bioimpedancia ha dejado huella sólida en el mercado deportivo y clínico, específicamente en los desarrollos y avances de la técnica, abren la puerta a futuros dispositivos portables con precisión clínica siendo, a su vez, menos invasivos [8].

Otro estudio inicia la relación entre la composición bioquímica celular y su posible uso para la temprana detección del cáncer, debido a que el cuerpo humano está compuesto por un alto porcentaje de solución electrolítica acuosa y se puede modelar, así como, futuramente, detectar cambios mínimos en los canales y flujos iónicos del cuerpo humano [9]. Además, diversa investigación ha brindado resultados optimistas en la utilización de la bioimpedancia para el estudio de las fracturas óseas y la recuperación de estas mediante la simplicidad de la obtención de datos de la misma técnica [10]. En general, el desarrollo de la bioimpedancia ha llegado a límites clínicos donde puede brindar un alto nivel de confianza a un bajo costo, no dañino para la integridad humana, poco tiempo de uso para obtener los resultados y altamente sensible a cambios leves de impedancia [11]. Basándose en esos principios, se planteó el desarrollo de un bioimpedancímetro portable con precisión clínica, el cual evaluará los siguientes datos fisiológicos mediante un análisis de bioimpedancia (BIA):

- Volumen de agua extracelular

- Volumen de agua intracelular

- Volumen de agua total

- Volumen de agua en exceso o déficit

- Masa celular total

- Masa del tejido adiposo

- Masa del tejido magro

- Masa de grasa

- Masa libre de grasa

- Masa muscular 


\section{Materiales y métodos}

La selección de la metodología se basa en el modelo Divergente-Convergente de Peter Ogrodnik para el diseño de dispositivos médicos en [12], el cual se caracteriza por cinco etapas en las que se contempla desde el recibimiento de la necesidad hasta el desarrollo del producto final. En la metodología planteada por Ogrodnik [12], la sección que converge es selectiva e inflexible, debido a la alta cantidad de procedimientos en el diseño general de dispositivos médicos. En [13] se explora la cantidad de variables involucradas en dispositivos médicos móviles, lo que levanta la discusión para un diseño centrado en el usuario más flexible (específicamente en el campo de los dispositivos portables) y un continuo testeo a lo largo de su desarrollo. En adición, [13] describe los distintos aspectos involucrados en el diseño de productos con precisión clínica que están en constante movimiento. Debido a estos factores, se procede a la unión con una metodología versátil como lo es el proceso proyectual [14], donde una constante iteración centrada en el usuario es requerida debido a la naturaleza del proyecto.

La metodología general de esta investigación llamada Divergente-Convergente Proyectual (DGC) se visualiza en la Figura 1.

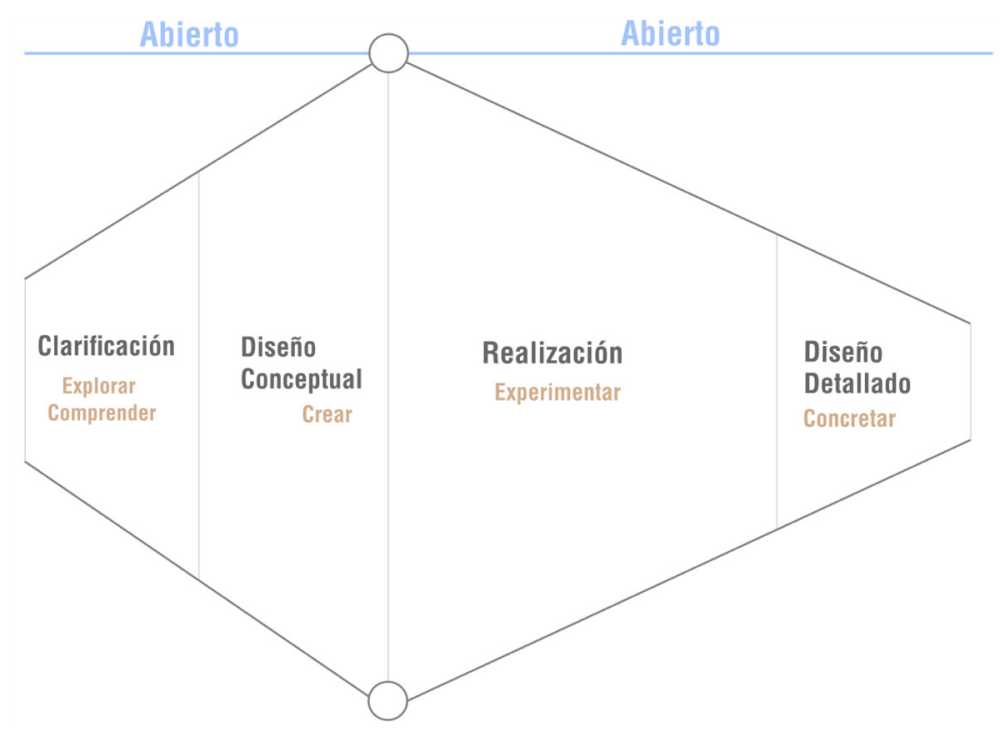

Figura 1. Estructura de la metodología Divergente-Convergente Proyectual Fuente. Elaboración Propia, 2020.

En donde las cinco etapas respectivas son:

1. Clarificación: etapa inicial en la que se procede a la revisión de aplicaciones previas para necesidades similares, así como el estudio y comprensión de los aspectos técnicos que posee la necesidad. Esta etapa recibe todos los antecedentes del proyecto por desarrollar, se dividen los requerimientos según sea la importancia respectiva y se procede con elaborar posibles soluciones a la necesidad. En esta etapa, se depura la necesidad a un campo más específico. 
2. Diseño conceptual: etapa en la que se establece la solución propuesta bajo las necesidades presentadas previamente. Se desarrollan prototipos digitales, visuales y de software de baja y mediana fidelidad con el objetivo de analizar el comportamiento del usuario.

3. Realización: desarrollo del prototipo y prueba con el mismo para verificar las variables involucradas. Se estudia mediante soluciones de alta fidelidad, el desempeño de las variables técnicas necesarias para cumplir con los requerimientos establecidos.

4. Diseño detallado: el proyecto por desarrollar se documenta y justifica previo a la norma requerida para generar su entrada al mercado. Todos los aspectos técnicos se preparan y se entregan a las partes involucradas con el objetivo de verificar las decisiones de diseño ejecutadas.

El proceso inicia con la lectura de la teoría respectiva y luego la recepción del prototipo previo desarrollado por el Grupo de Ingeniería Biomédica perteneciente a la Universidad de Sevilla, así como las especificaciones técnicas necesarias para la optimización de este en sus excesivas dimensiones tridimensionales, interfaz y adaptabilidad con el usuario, accesibilidad de técnica, así como de interacción. Seguidamente, se realizan visitas de campo con los involucrados en el uso y desarrollo del producto; es desarrollado un estudio de la cuestión cualitativo mediante entrevistas con el personal involucrado en el proyecto, así como los involucrados del mismo. En adición, se visitó la Asociación de Pacientes con Afección Renal Crónica (Alcer Giralda) de Sevilla, España, y se realizaron entrevistas cualitativas para conocer el impacto psicológico del uso del producto en la enfermedad renal crónica.

Además, se realizaron entrevistas cualitativas con los ingenieros especialistas en Telecomunicaciones y Modelo de Señales para comprender los requisitos técnicos que debía cumplir el diseño. Consecutivamente, se establece una lista de posibles prototipos que puedan cumplir los requisitos establecidos, ergonómicamente justificados en [15] y se depuran para obtener uno solo, el cual se construye, se ensambla y se comprueba con usuarios finales. Ensamblado el producto, se realiza la demás documentación de apoyo basado en la normativa CE bajo la directriz 93/42/EEC [16], análisis de costos y guías técnicas de soporte para el futuro desarrollo masivo del producto.

\section{Herramientas de desarrollo del producto}

Para el desarrollo del producto se utilizaron herramientas de Diseño Asistido por Computadora como SolidWorks, Fusion 360 y entre las herramientas específicas se utilizó SolidWorks Plastics, SolidWorks Costing y Fusion 360 Surface Modelling. Se requirió un fuerte desarrollo de co-diseño con elementos pre estandarizados para ajustar el diseño de la carcasa con las mejores prestaciones de adaptabilidad. 


\section{Herramienta de prototipado}

Los prototipos generados se fabricaron en impresión 3D con PLA como material base y el posterior acabado fue brindado para explorar las distintas visualizaciones físicas del producto. La impresora que se utilizó fue una Prusa Research Prusa3D.

\section{Resultados}

El rediseño de la usabilidad ergonómica fue el primer factor más importante para generar un dispositivo accesible a la mayor cantidad de población posible y, específicamente, se consideró la necesidad de los pacientes con enfermedad renal crónica, los cuales poseen una movilidad limitada para colocarse el producto. Los parámetros ergonómicos del producto se establecen con las tablas antropométricas establecidas en [15].

Las medidas requeridas para el desarrollo de la carcasa y el tamaño de los electrodos del dispositivo fueron las siguientes:

- Largo de la mano (186.3 mm)

- Largo de la palma (110.1 mm)

- Ancho de la mano (83 $\mathrm{mm})$

- Largo del dedo índice (74.7 mm)

- Ancho del dedo índice $(21 \mathrm{~mm})$

- Ancho del pie $(98.9 \mathrm{~mm})$

- Largo del pie $(260.5 \mathrm{~mm})$

La selección de los percentiles corresponde al 65\%, debido a que la condicionante electrónica del dispositivo requiere un tamaño de carcasa balanceado y los electrodos, los cuales se deben colocar en la palma del pie y de la mano del usuario, deben cubrir la mayor cantidad de área sin generar algún tipo de incomodidad en el usuario [1].

Las medidas requeridas para el desarrollo del cableado del dispositivo fueron las siguientes:

- Estatura (1712 mm)

- Altura puño-pie (754 mm)

- Ancho de hombros (436 mm)

Debido a que el dispositivo está limitado por el uso del cableado, se establece que un percentil del $95 \%$ de los valores mencionados previamente son los más adecuados para cubrir a toda la población y facilitar el alcance entre usuario-dispositivo.

La justificación de la navegación física se desarrolla bajo las normas ISO 9995-7 (Keyboard 
layouts for text and office systems -Part 7: Symbols used to represent functions) y la norma IEC 60417 (Graphical symbols) [17]. En adición, la selección de una norma estandarizada se escoge debido a la amplia cantidad de usuarios que utilizarán el dispositivo, lo cual facilita, a su vez, la navegación en la interfaz física. Los íconos seleccionados fueron comprobados mediante pruebas de usabilidad en usuarios reales con edades entre los 25 a los 64 años en pruebas aleatorias, con el objetivo de determinar el tamaño, relieve y composición de la interfaz física.

Debido a que el dispositivo requiere de funciones gráficas tales como:

- Aceptar

- Retornar

- Subir

- Bajar

La norma establece los gráficos específicos para cada función mediante un símbolo estandarizado que puede indicarse posteriormente en la guía técnica del producto y así facilitar los futuros procesos de manufactura y productivos. La Figura 2 y Figura 3 describen el tipo de símbolo por cada función.

\begin{tabular}{|c|c|c|c|c|}
\hline $\begin{array}{l}\text { ISO/IEC 9995-7 } \\
\text { Número }\end{array}$ & $\begin{array}{l}\text { ISO } 7000 \\
\text { IEC } 4177 \\
\text { Número }\end{array}$ & Símbolo & Función & Descripción \\
\hline 24 & ISO $7000-1025$ & & Aceptar & $\begin{array}{l}\text { Enviar datos o mensajes } \\
\text { a una aplicación determinada }\end{array}$ \\
\hline 30 & ISO 7000-2106 & $\stackrel{\circ}{0}$ & Deshacer & $\begin{array}{l}\text { Retornar a un estado previo } \\
\text { del que se encuentra }\end{array}$ \\
\hline 31 & IEC 417-5107 & $\Delta$ & Arriba & Mover el cursor hacia arriba \\
\hline 32 & IEC 417-5107 & 古 & Abajo & Mover el cursor hacia abajo \\
\hline
\end{tabular}

Figura 2. Funciones de navegación estandarizadas

Fuente. Elaboración propia, 2020. 
La función de apagado y encendido debe ir justificada bajo la norma de equipos electrónicos IEC 60417 por lo que se procede a establecer el símbolo asignado para la función EncenderApagar.

ISO/IEC 9995-7
$\begin{aligned} & \text { ISO } 7000 \\ & \text { Número }\end{aligned}$
Número

Figura 3. Función de apagado y encendido estandarizada

Fuente. Elaboración propia, 2020.

\section{Sistema de encapsulamiento principal}

El encapsulamiento principal se resolvió mediante un diseño simple e intuitivo. Los cuatro botones principales presentaron el color gris, debido a que los usuarios requirieron alto contraste para diferenciar el indicador de la función. A su vez, el sistema de encendido se realizó mediante la acción del botón aceptar y el apagado se genera automáticamente (Figura 4).

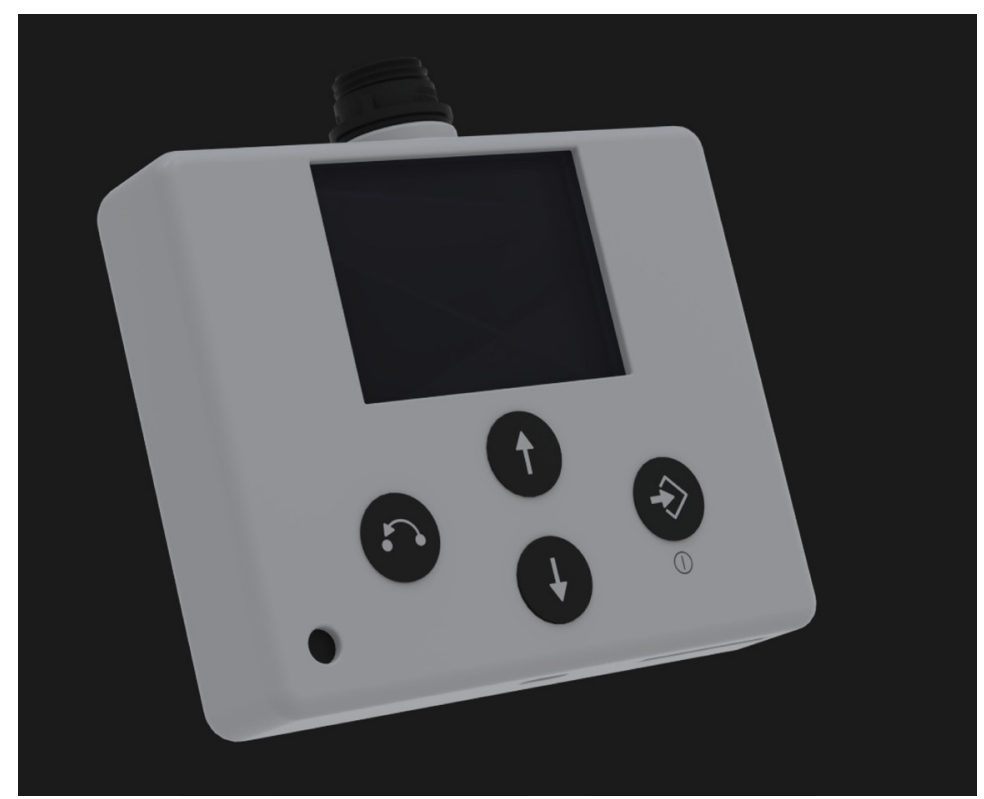

Figura 4. Encapsulamiento principal

Fuente. Elaboración propia, 2020. 
Los indicadores de las entradas USB y SD se declararon textualmente con el objetivo de facilitar su ubicación y lectura (Figura 5).

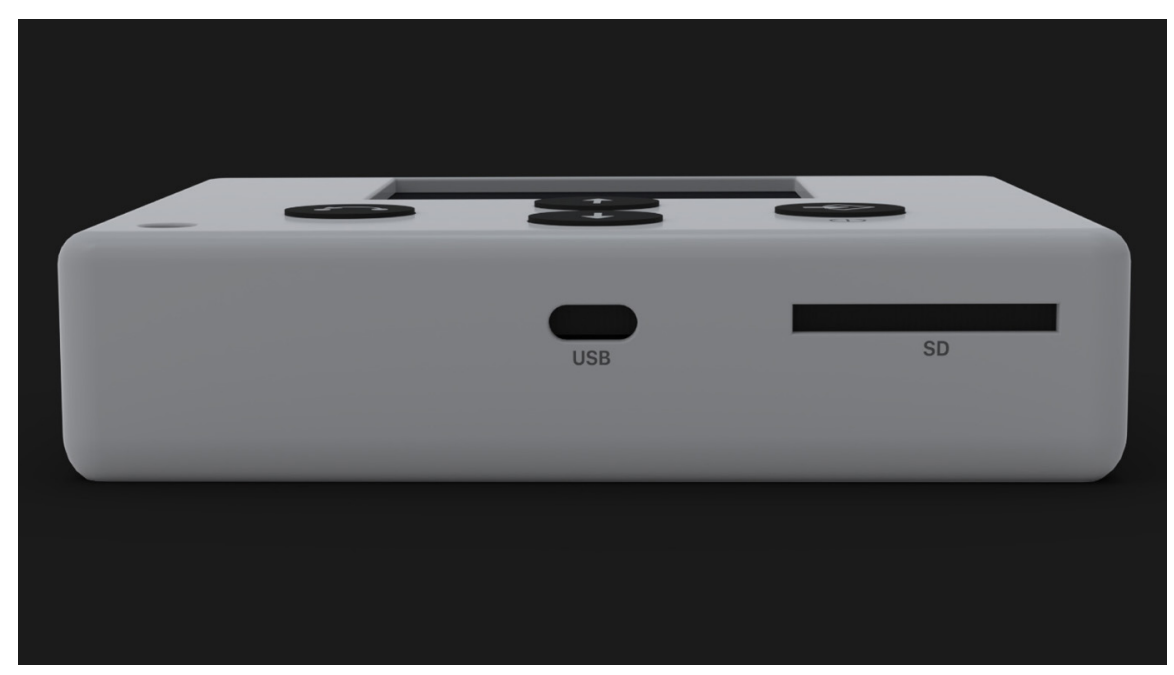

Figura 5. Detallado de la especificación de las conexiones

Fuente. Elaboración propia, 2020.

En el caso de la topología del dispositivo, los botones se presentan elevados para facilitar su ubicación en el espacio para los usuarios. La conexión de bioimpedancia se eleva de la carcasa, debido a que debe existir un espacio de seguridad entre la PCB y la conexión, con el objetivo de evitar problemas de medición por fallos en el ensamblaje. En adición, la conexión USB (cargar el dispositivo) y la conexión SD (resguardo de datos) se agrupan juntas en la parte inferior del dispositivo con el objetivo de agrupar su función mediante la jerarquía del funcionamiento (Figura 6).

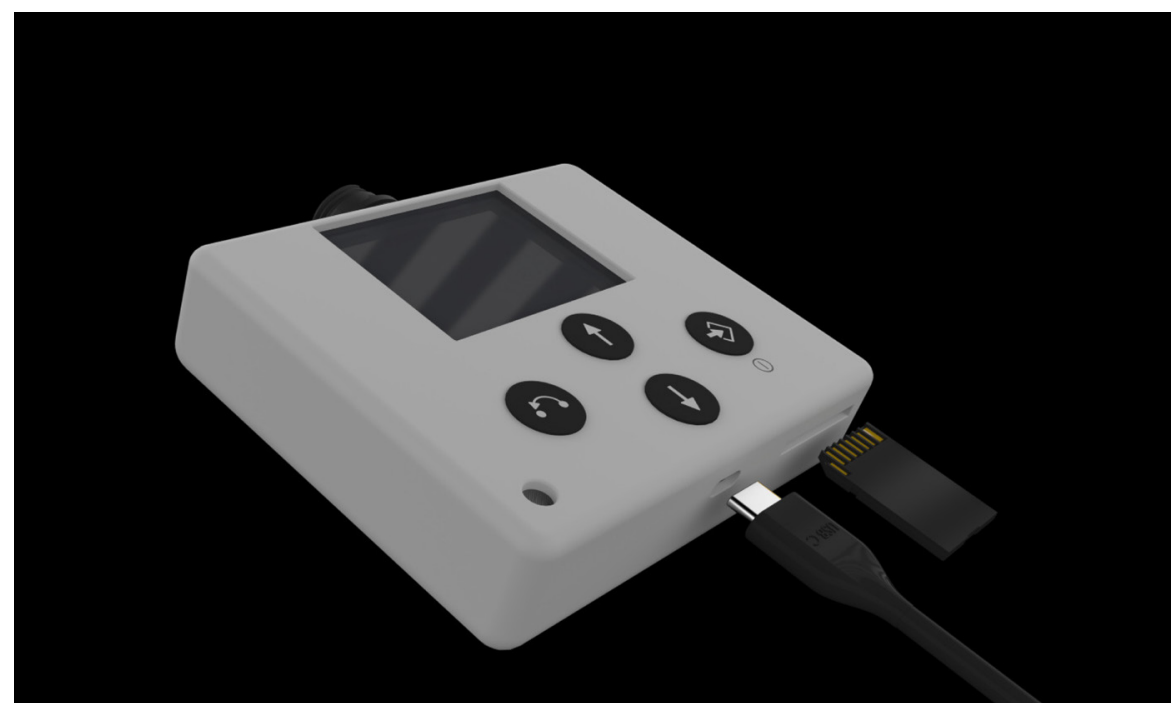

Figura 6. Conexiones de comunicación y de alimentación

Fuente. Elaboración propia, 2020. 
La conexión de bioimpedancia, por requerimiento técnico justificado con entrevistas con expertos, debe estar aislada de las conexiones auxiliares del dispositivo. En su caso, la conexión USB (cargar el dispositivo) y la conexión SD (resguardo de datos) se agruparon juntas en la parte inferior del dispositivo, con el objetivo de unificar su función mediante la jerarquía del funcionamiento.

En adición, la conexión de bioimpedancia se elevó de la carcasa, debido a que debe existir un espacio de seguridad entre la PCB y la conexión, con el objetivo de evitar problemas de medición por fallos en el ensamblaje. El conector de bioimpedancia está lo más cerca de la superficie inferior, con el objetivo de mantener la posición horizontal, tal como se muestran en la Figura 7. Así mismo, el sistema de acople presenta un leve giro de roscado con el objetivo de evitar la entrada de polvo o agua a los contactos y el cableado interno viene protegido por una funda de poliéster sellada mediante compresión con el conector.

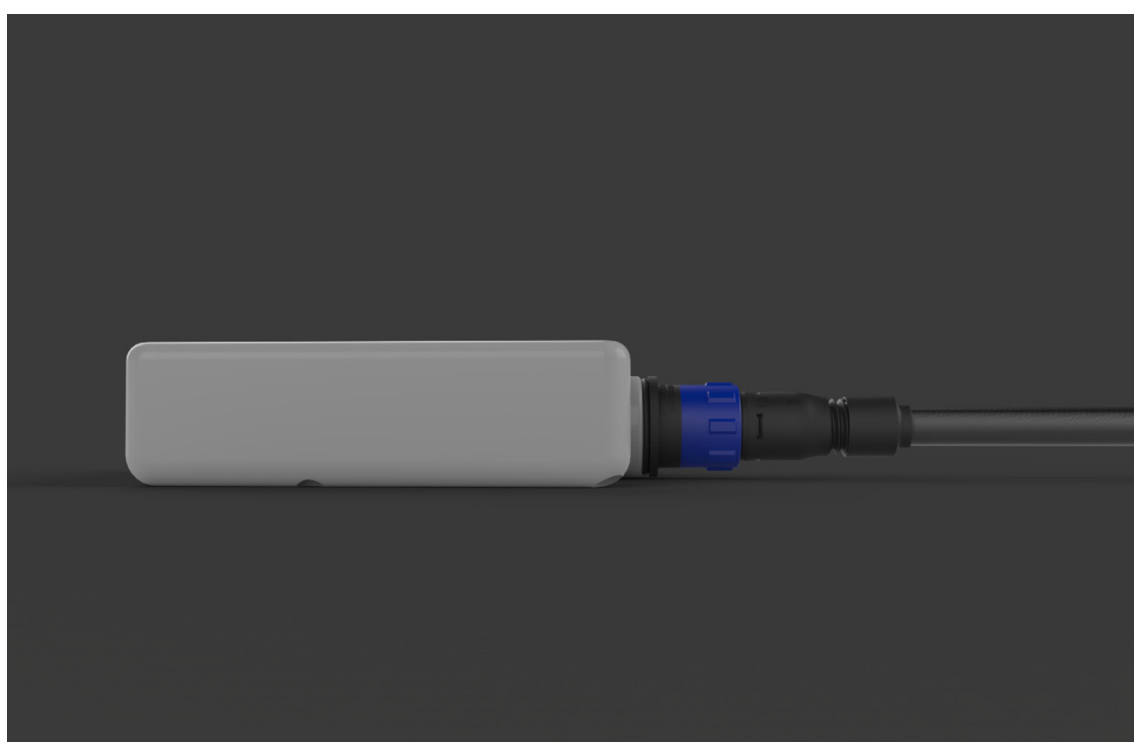

Figura 7. Conexión de bioimpedancia

Fuente. Elaboración propia, 2020.

El cable de bioimpedancia (Figura 8) en su totalidad tiene 2 metros de largo divididos en un metro de cableado unido y cada conexión saliente posee otro metro. Los valores se establecieron mediante el análisis antropométrico de la población en [15]. En adición, el recubrimiento de la conexión se establece mediante poliéster comprimido para evitar el desarrollo de nudos y la afectación interna del cableado. La importancia del aislamiento con el exterior se debe a que la conexión de bioimpedancia puede ser manipulada por agentes externos y alterar la medición [1]. 


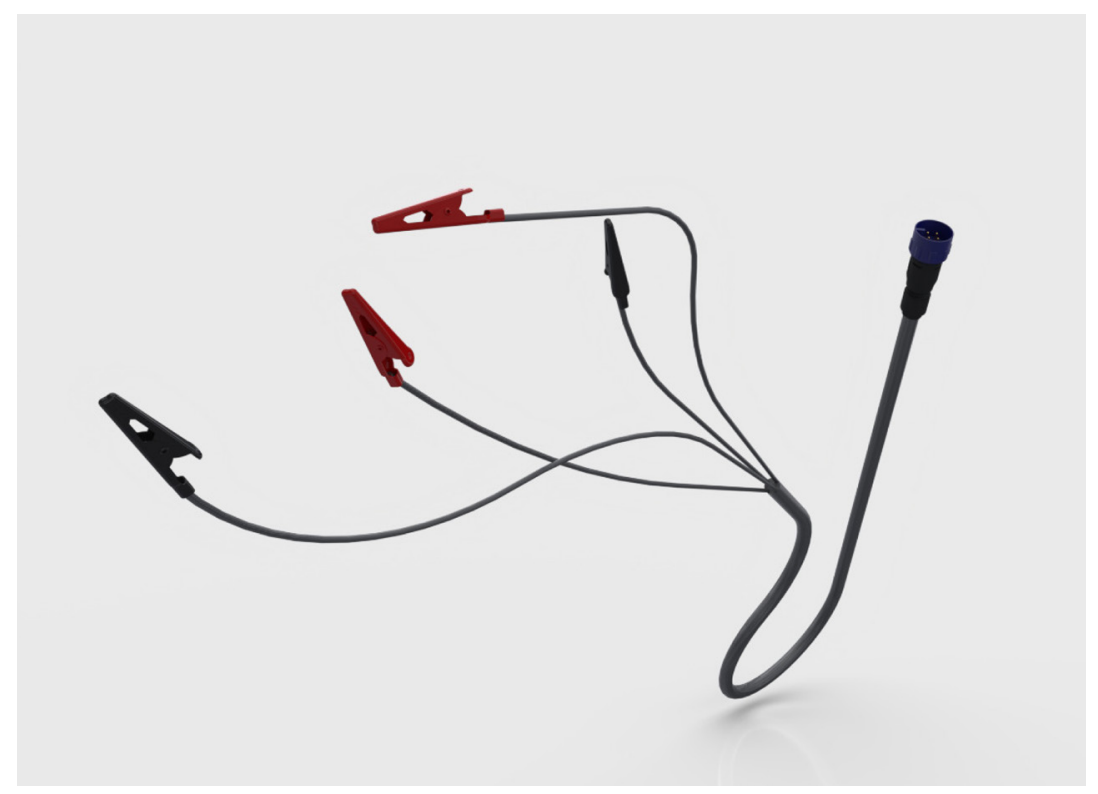

Figura 8. Cableado general

Fuente. Elaboración propia, 2020.

El prototipo de alta fidelidad (Figura 9) presenta las conexiones exactas e indicadas en los requerimientos para así poder validar su funcionamiento de una manera más precisa mediante los parámetros técnicos establecidos previamente [16]. En adición, se construyó mediante manufactura aditiva en una impresora Pruza Research del laboratorio del Grupo de Ingeniería Biomédica. Los demás componentes fueron ordenados a las casas matrices correspondientes para así ensamblar de manera exitosa.

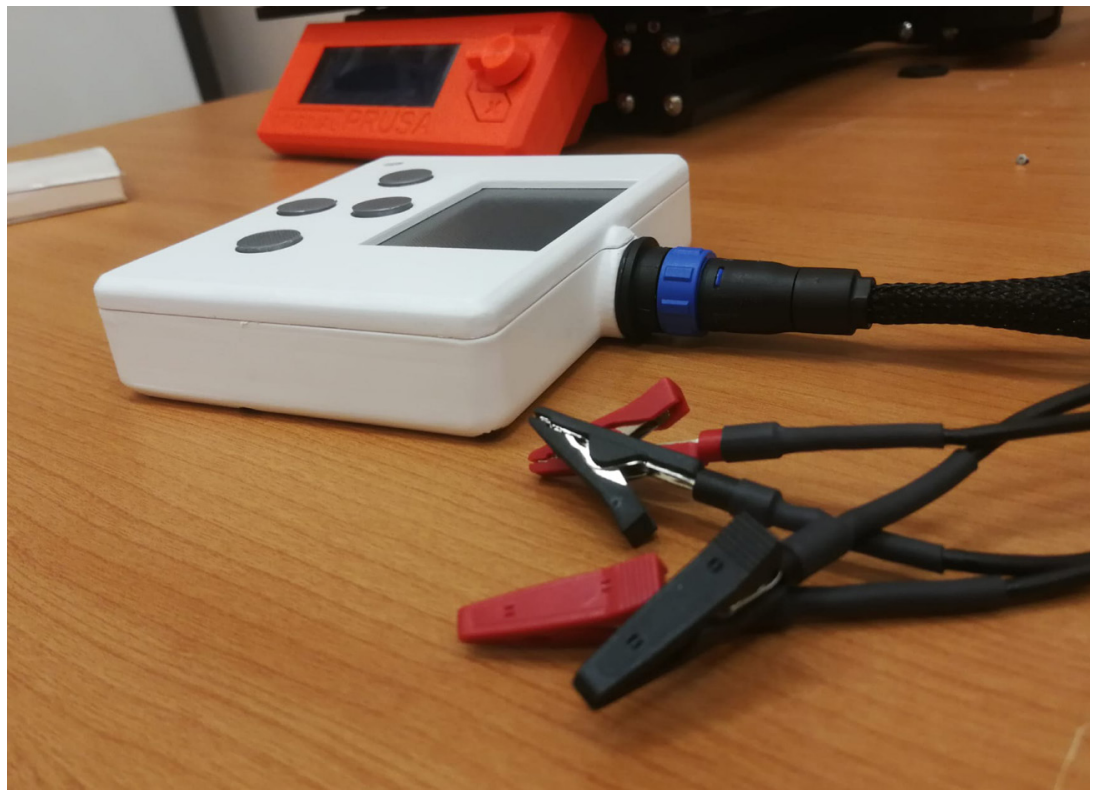

Figura 9. Prototipo de alta fidelidad

Fuente. Elaboración propia, 2020. 


\section{Discusión de resultados}

El prototipo elaborado presenta un diseño industrial viable para el desarrollo de un bioimpedancímetro portable, sin embargo, una verificación con usuarios de manera masiva en entornos hospitalarios y domésticos es recomendada para mejorar la iteración del producto. El prototipo desarrollado presenta una solución unificada en comparación con [18] y [19]. La metodología DGC es un principio novedoso, sin embargo, se requiere futura aplicación y revisión por pares internacionales, así como una inclusión en proyectos por desarrollar en el campo de la telemedicina y desarrollo de productos de monitoreo portables. Es aconsejable generar una segunda revisión con expertos en el campo del diseño, sobre la normativa escogida para preparar el producto con la normativa seleccionada. En adición, se recomienda realizar pruebas clínicas para evaluar el desempeño de la obtención de los datos de bioimpedancia en el prototipo desarrollado.

\section{Conclusiones}

El diseño del bioimpedancímetro presenta una aproximación novedosa y justificada a nivel de usuario [11]. El trabajo en la teoría fue uno de los pilares principales, debido a que el diseño de la interfaz física de un dispositivo clínico va estrechamente relacionado con la función de este, por lo que una metodología novedosa fue aplicada para el desarrollo de este [12]. La línea de involucrados es, a su vez, un aspecto que desde el inicio se tuvo en cuenta en el desarrollo de este trabajo siguiendo a [13]. Las decisiones del diseño del dispositivo van estrechamente relacionadas con la observación, la visita y entrevista con pacientes reales en la Asociación Alcer Giralda de Sevilla. Esto a su vez, establece un futuro usuario del dispositivo en un escenario real.

Se determinó un diseño manufacturable justificado mediante costos y materiales escogidos [20], así como con la justificación de esclarecimiento jurídico que viene detrás del desarrollo del dispositivo médico; específicamente con la aprobación de entrada en el mercado del marco de Comunidad Europea [16]. En adición, el diseño fue producto de un trabajo multidisciplinario donde el diseñador tuvo la responsabilidad de realizar una lectura previa sobre el diseño electrónico para así colaborar en las mejoras de la placa establecida, con el fin de generar un diseño integrado que brinde las mejores prestaciones. Además, otro aspecto que facilita el cómodo uso del dispositivo fue la gradiente de mejora en el cableado de bioimpedancia y medidas antropométricas, estableciendo un largo de dos metros con un metro de separación por conexión calculado con base en [15].

La metodología DGC presenta una aproximación novedosa al desarrollo de proyectos médicos multidisciplinarios, sin embargo, una exploración más profunda es recomendada y bienvenida. 


\section{Recomendaciones}

Para el futuro desarrollo del dispositivo, se recomienda la validación a nivel electrónico y de la programación computacional con respecto a las directrices de la Comunidad Europea. A su vez, la construcción final de diversos componentes para su ensamblaje y el respectivo ajuste a la carcasa establecida. Así mismo, pruebas clínicas del diseño final del dispositivo en entornos clínicos son recomendables como herramienta de mejora continua.

\section{Reconocimientos}

La investigación fue realizada bajo el financiamiento de la Beca de Pregrado de Investigación por medio del Consejo Nacional de Rectores bajo el memorando (N.º6-2016) de Aprobación y postulación de la Escuela de Ingeniería en Diseño Industrial y en concordancia con el Grupo de Ingeniería Biomédica código PAIDI-TIC203 perteneciente a la Escuela Técnica de Ingenierías de la Universidad de Sevilla.

\section{Referencias}

[1] Naranjo-Hernández D, Reina-Tosina J, Buendía R, Min M, Bioimpedance Sensors: Instrumentation, Models, and Applications, Journal of Sensors, vol. 2019, Article ID 5078209, 2 pages, 2019. https://doi.org/10.1155/2019/5078209

[2] Wahezi SE, Kohan LR, Spektor B, Brancolini S, Emerick T, Fronterhouse JM,Luedi M, Colon MA, Kitei PM, Anitescu M, Goeders NE, Patil S, Siddaiah H, Cornett EM, Urman RD,Kaye AD, Telemedicine and Current Clinical Practice Trends in a COVID-19 Pandemic, Best Practice \&Research Clinical Anaesthesiology, https://doi.org/10.1016/j.bpa.2020.11.005.

[3] Weiner S, Fink J,Telemedicine to Promote Patient Safety: Use of Phone-Based Interactive Voice-Response System to Reduce Adverse Safety Events in Pre-dialysis CKD,Advances in Chronic Kidney Disease,Volume 24, Issue 1,2017,Pages 31-38,ISSN 1548-5595,https://doi.org/10.1053/j.ackd.2016.12.004.

[4] Jha V, Garcia-Garcia G, Iseki K, Chronic kidney disease: global dimension and perspectives. Lancet. 2013

[5] M. A. Estudillo-Valderrama et al., "A Distributed Approach to Alarm Management in Chronic Kidney Disease," in IEEE Journal of Biomedical and Health Informatics, vol. 18, no. 6, pp. 1796-1803, Nov. 2014, doi: 10.1109/JBHI.2014.2333880.

[6] Bonuccelli A, Marzatico F, Stesina G, Stefanini L, Buonocore D, Rucci S, Tencone F, Gatteschi L, Bioelectrical impedance vector analysis (BIVA) to evaluate seasonal variations in body composition of elite soccer players. Journal of Sports Nutrition. 2011

[7] Ling C, De Craen A, Slagboom P, Gunn D, Stokkel M, Westendorp R, Maier A, Accuracy of direct segmental multi-frequency bioimpedance analysis in the assessment of total body and segmental body composition in middle-aged adult population,Clinical 


\section{revistaIDI+}

Nutrition,Volume 30, Issue 5,2011,Pages 610-615,ISSN 0261-5614, https://doi. org/10.1016/j.clnu.2011.04.001.(http://www.sciencedirect.com/science/article/pii/ S0261561411000665)

[8] Fernández-Corazza M, Turovets S, Luu P, Anderson E, Tucker D, Transcranial Electrical Neuromodulation Based on the Reciprocity Principle. Frontiers in Psychiatry. 2016

[9] Herrera L, Chaparro C, Pinto S, Corzo S, Torres-Mejias M, Electrical Properties of Normal Cervical Human Cells in Suspension: The Relation between Normal Tissue and Electrical Impedance Spectrum. Springer Link. 2016

[10] Dell'Osa H, Concu F, Dobarro J, Felice C, Bone Fracture Detection by Electrical Bioimpedance: First Non-Invasive Measurements in Ex-Vivo Mammalian Femur. BioRxiv. 2019

[11] Demers G, Migliore S, Bennett DR, Ultrasound evaluation of cranial and long bone fractures in a cadaver model. Mil Med. 2012.

[12] Ogrodnik P, Medical Device Design. Elsevier. 2013.

[13] Stein R, Wilklund M, Human Factors in Medical Device Design, CRC Press. Pages 715747. 2011

[14] Araya-Rojas L, Granados-Gómez D, Experiencia de aplicación del design thinking al curso de métodos de desarrollo de productos. CIMTED. 2016

[15] Matzdorff J, Windberg J. International Anthropometric Data for Work-Place and Machinary Design. Federal Ministry of Labour and Social Affairs. 1998

[16] Council of European Communities, COUNCIL DIRECTIVE 93/42/EEC, Official Journal of the EuropeanCommunities,1993.

[17] International Electrotechnical Commission, Symboles graphiques utilisables sur le matériel. Graphical symbols for use on equipment. ISO. 2015.

[18] Villa F, Magnani A, Castiglioni P, Portable Body Impedance System for Long-Term Monitoring of Body Hydration, International Conference on Pattern Recognition, Informatics and Medical Engineering, PRIME, 1-4, 2012.

[19] Corbellini S, Vallan A, Arduino-based portable system for bioelectrical impedance measurement, IEEE MeMeA 2014 - IEEE International Symposium on Medical Measurements and Applications.

[20] Goodship V, Middleton B, Cherrington R, Design and manufacture of plastic components for multifunctionality: structural composites, injection molding, and 3D printing. Elsevier.2016.

[21] Naranjo-Hernández D, Reina-Tosina J, Min M, Fundamentals, Recent Advances, and Future Challenges in Bioimpedance Devices for Healthcare Applications", Journal of Sensors, vol. 2019, Article ID 9210258, 42 pages, 2019. https://doi. org/10.1155/2019/9210258 